Petra Storjohann*

\title{
Dynamischer Gebrauch von Paronymen in allgemein-, fach-, wissenschafts- und bildungssprachlicher Kommunikation
}

https://doi.org/10.1515/zfal-2019-2018

Abstract: This paper focuses on easily confused items (so-called paronyms) in German in terms of their general, technical or academic contextual uses. It outlines the semantic discrepancies between contextual usages of pairs such as $M e$ thode/Methodologie/Methodik and unehelich/nichtehelich/außerehelich depending on their linguistic registers and varieties. While previous studies lack empirical evidence and primarily operate with morphological criteria (cf. Lăzărescu 1999) the descriptions here derive from corpus-based examinations of general written and of technical discourse. It is shown that causes of lexical confusion arise from formal, phonetic resemblances or semantic similarities, regular co-occurrence, incorrect morphological analogies and political governance of language. Context, knowledge, associations and experience determine the choice of lexical terms. Speakers need to apply linguistic and extra-linguistic principles in order to create adequate contexts. With the help of paronym examples and corpus data, these will be elucidated in more detail.

Keywords: Paronyms, Commonly Confused Words, Lexical Confusion, General/ Technical/Academic Usage

\section{Einleitung}

Unter Paronymie wird die Verwechselbarkeit von formal, lautlich und/oder semantisch ähnlichen Ausdrücken verstanden, die häufig zusätzlich über gemeinsame morphologische Wurzeln verfügen (Lăzărescu 1999). Sie gehören zu den typischen sprachlichen Zweifelsfällen, die bei Muttersprachler*innen wie Fremdsprachenlernenden Unsicherheiten verursachen können. Es handelt sich hierbei um ein heterogeneres und größeres Phänomen als ursprünglich gedacht (siehe

*Korrespondenzautor: Petra Storjohann, Leibniz-Institut für Deutsche Sprache, R 5, 6-13, 68161 Mannheim, Deutschland, E-Mail: storjohann@ids-mannheim.de 
Schnörch 2015). ${ }^{1}$ Ca. 2.000 Paare lassen sich grob als Paronyme der deutschen Gegenwartssprache bezeichnen. Bei genauerer Betrachtung zeigt sich, wie grobkörnig die oben genannte Begriffsbestimmung des Phänomens ist, und dass der kontextuelle Gebrauch von Paronymen sehr unterschiedliche Ausprägungen aufweisen kann. So gibt es Paare, die vollständig unterschiedlich gebraucht werden (z.B. legal/legitim). Es gibt Paare/Gruppen, die vollständig synonym sind, bei denen Sprecher*innen allerdings unsicher sind, ob die jeweiligen Gebrauchsbedingungen nicht doch in semantischen Feinheiten voneinander abweichen (z.B. patriarchalisch/patriarchal/patriarchisch, Adaption/Adaptation). Manchmal weisen Paronyme sowohl deutliche semantische Gemeinsamkeiten also auch Unterschiede auf (z.B. sportlich/sportiv, musikalisch/musisch) oder sie verfügen in speziellen Diskursen über semantische Nuancen (unsozial/asozial/antisozial). Manche dieser Ausdrücke werden gelegentlich vertauscht, beim Gebrauch anderer gibt es große Zweifel, wieder andere verursachen keinerlei Unsicherheiten.

Noch komplexer wird das Phänomen der Paronymie, wenn man verwechselbare Ausdrücke im allgemeinen öffentlichen Sprachgebrauch mit Verwendungen in der Fach- (z.B. Parodontitis/Parodontose) und Wissenschaftssprache (z.B. Methode/Methodologie/Methodik, Evaluation/Evaluierung) vergleicht. So können die Bedeutungen paronymer Ausdrücke in der Allgemeinsprache und in der fachlichen Kommunikation z.T. voneinander abweichen. Auch aus der Verwaltungssprache kommen Paronyme (unehelich/nichtehelich/außerehelich), die zu Unsicherheiten in Alltagsgesprächen führen. Diese Paare/Gruppen zeigen, dass die Paronymie ein dynamisches Phänomen ist, das Veränderungen unterliegt. Diese sind auch in diachroner Hinsicht zu beobachten (vgl. sensibel/sensitiv in Storjohann 2015). In mündlicher Kommunikation etwa kommt es schneller zu fehlerhaften Verwendungen als z.B. in redigierten Texten. Vor allem in speziellen Kontexten zeigt sich, dass angemessener Gebrauch nicht ausschließlich von lexikalischem, sondern vielmehr auch von außersprachlichem Wissen sowie vom Grad der sprachlichen Erfahrung abhängt. Ähnlich klingende, geschriebene oder verwendete Ausdrücke können sich gegenseitig semantisch beeinflussen, eine anfänglich noch als falsch empfundene Verwendungsweise kann sich verfestigen und das kontinuierliche Verwechseln kann Auswirkungen auf semantische Entwicklungen haben. Sprachliche Rivalität kann für das Verdrängen bestimmter Formen sorgen oder die Verwendung semantisch ähnlicher Ausdrücke kann eines

1 Unsicherheiten bestehen bei Muttersprachler^innen gleichermaßen wie bei Fremdsprachenlerner`innen, allerdings variieren diese zwischen diesen beiden Gruppen und es gibt zusätzlich, je nach Ausgangssprache der Fremdsprachenlerner^innen, auch noch verschiedenartige Unsicherheiten bei Übersetzungen. 
der beiden Paronyme zu einer stärkeren semantischen Ausdifferenzierung drängen, um für neuerliche Abgrenzung zu sorgen.

Warum manche Paronyme stärker verwechselt werden als andere und welche Ursachen dahinterliegen, ist in der lexikalischen Semantik bisher wenig erforscht. Unter strukturalistischer Betrachtung spielten Faktoren wie Abweichung, Dynamik, kontextuelle Veränderbarkeit oder diskursive Anpassung keine Rolle. Die traditionelle, rein formalistische Beschreibung Lăzărescus (1995, 1999), bei der die Paronymie aus Sicht des Fremdsprachenunterrichts als potentielle Fehlerquelle verstanden wurde, berücksichtigte beispielsweise weder die vielfältigen Ausprägungen und Entwicklungstendenzen noch variable Kontextualisierung. Vielmehr strebte sie nach einem umfassenden Klassifikationsmodell, das lexikalische Zweifelsfälle primär nach morpho-syntaktischen Eigenschaften gruppierte. Das Zustandekommen von Verwechslung oder Auswirkungen von regelhafter Verwechslung auf semantische Prozesse wurde bei der Erarbeitung strukturalistischer Typologien nicht hinterfragt. Auch semantische Beschreibungen des Phänomens auf der Grundlage von Befunden des Sprachgebrauchs blieben lange unberücksichtigt. Genau hier knüpft der vorliegende Beitrag an.

Dieser Beitrag widmet sich der Paronymie aus korpussemantischer Sicht unter Berücksichtigung kognitiver Prinzipien. Ausgangspunkt paronymer Zweifelsfälle sind nicht formale, meist morphologische Unterschiede, sondern das Paronyme verbindende Element, die potenzielle Verwechselbarkeit und die Analyse ihres kontextuellen Gebrauchs. Erste korpusbasierte Annäherungen an dieses lexikalische, aber auch kognitive Phänomen aus muttersprachlicher Sicht legen Mell/Schnörch/Storjohann (2019) für Paronyme in der öffentlichen schriftsprachlichen Kommunikation vor. Im Vordergrund stehen dabei diskursiv-kommunikative Auffälligkeiten des kontextuellen Gebrauchs. Nachfolgend werden einige Spezialfälle der Paronymie aufgezeigt, bei denen sprachliche Vielfalt und kontextuelle Anpassung im gegenwärtigen Gebrauch und in verschiedenen Varietäten beobachtbar sind. Besonders auffällig ist dabei die Variation zwischen allgemeinem Sprachgebrauch und solchem in der Fach-, Bildungs-, (allgemeinen) Wissenschafts- oder Verwaltungssprache. Hier sollen die dynamischen Prozesse aufgezeigt werden, in denen Paronyme verschiedene semantische Muster eingehen. Denn genau diese Dynamik verursacht Verständigungsschwierigkeiten in der fachsprachlichen Kommunikation oder Schwierigkeiten bei der Sprachvermittlung. Andererseits lösen solche Differenzen auch semantische Prozesse aus, die die Bedeutung der Paronyme verändern können. Zuletzt sollen auch mögliche Ursachen und die unterschiedlichen Beziehungen zwischen den einzelnen Paronympartnern näher betrachtet werden, um der Komplexität und Vielfältigkeit des Phänomens näher zu kommen. 
Die Studien, auf deren Grundlage nachfolgende Erkenntnisse beruhen, erfolgten im Rahmen des Projektes „Paronymwörterbuch“ am Leibniz-Institut für Deutsche Sprache in Mannheim, dem ersten korpusgestützten, gegenwartssprachlichen Nachschlagewerk für leicht verwechselbare Ausdrücke des Deutschen. ${ }^{2}$ Alle aufgeführten Beispiele sind dort dokumentiert.

\section{Datengrundlage}

Die umfassende empirische Untersuchung deutscher Paronyme im Gebrauch ist das Ziel des Projektes „Paronymwörterbuch“, das neben der lexikografischen Dokumentation auch lexikologischen Fragestellungen nachgeht. Zu diesem Zweck wurde ein spezielles Paronymkorpus aufgebaut. Zahlreiche theoretische Forschungsfragen ließen sich bereits auf der Basis dieses Materials beantworten. Aber auch metalexikografische Überlegungen bzw. Chancen und Probleme verschiedener methodischer Ansätze wurden bereits näher beleuchtet (vgl. Storjohann (Hrsg.) 2019). ${ }^{3}$ Neben einer Vielzahl an Textquellen und guter regionaler Ausgewogenheit bietet dieses Korpus vor allem den Vorteil, öffentlich zugänglich zu sein, sodass die lexikografische Arbeit am Wörterbuch „Paronyme - Dynamisch im Kontrast“ transparent und nachvollziehbar ist. Es enthält überwiegend journalistische Texte aus 27 regionalen und überregionalen Zeitungen sowie Zeitschriften aller Jahrgänge ab 1990. ${ }^{4}$ Neben Auswertungen mit dem Ziel, die Ausdrücke in der öffentlichen Kommunikation näher zu beleuchten, sollen ebenfalls fachsprachliche bzw. alltagswissenschaftliche Kommunikationssituationen erfasst werden. Um divergente Gebrauchsweisen von Paronymen gezielter auswerten zu können, vor allem bei fach- oder wissenschaftssprachlichen Verwendungen fremdsprachlicher Ausdrücke, wurden zusätzliche Quellen aus dem Deutschen Referenzkorpus (DeReKo) $)^{5}$ konsultiert, da dieses zusätzlich reine Fachsprachen-Subkorpora enthält. Auch spezielle Zeitschriften zu Fachbereichen wie etwa Gentechnologie, Geschichte, Computertechnik, Tourismus, Börse, Kochen oder Flugverkehr bzw. populärwissenschaftliche Zeitungen wie z.B. spektrumdirekt, Stern Gesund leben,

2 Das Wörterbuch ist kostenlos online zugänglich: https://www.owid.de/parowb.

3 Das Sonderheft enthält verschiedene Beiträge zu unterschiedlichen Schwerpunkten, die im Projekt zwischen 2015 und 2018 verfolgt wurden.

4 Das Korpus ist kostenlos (http://www1.ids-mannheim.de/lexik/paronymwoerterbuch/dasparo nymkorpus.html) und über das Korpusanalyse- und Recherchetool COSMAS II nutzbar: https://co smas2.ids-mannheim.de/cosmas2-web.

5 DeReKo = Deutsches Referenzkorpus: (http://www1.ids-mannheim.de/kl/projekte/korpora.ht $\mathrm{ml})$. 
Psychologie Heute oder P.M. Magazin sind darin enthalten und dienten als zusätzliche Informationsquellen.

Beide Korpora bildeten die Grundlage diverser quantitativer und qualitativer Untersuchungen des kontextuellen Umfeldes einzelner Paronympaare. Die Auswertung der verschiedenen Textquellen und die Heterogenität der Paronympaare machten es auch erforderlich, komplementäre Methoden mit jeweils unterschiedlichen Fokussierungen zu nutzen und verschiedene lexikalisch-semantische Aspekte auszuwerten. Eine entscheidende Rolle für die Bedeutungsanalyse eines Wortpaares spielen statistische Verfahren zur Erforschung kontextueller Umgebungen von Einzellexemen. Darüber hinaus kam auch eine bisher wenig genutzte Methode, nämlich ein kontrastives kartografisches Verfahren zur Ermittlung und Visualisierung semantischer Nähe zwischen zwei Lexemen zum Einsatz. Beide empirischen Ansätze, ihre jeweiligen Vor- und Nachteile, werden ausführlich und anhand von konkreten Beispielen in Schnörch (2019) und Teichmann (2019) diskutiert.

\section{Allgemein- und fachsprachliche Paronyme}

Unter allgemeinsprachlichen Paronymen werden in diesem Beitrag solche Ausdrücke verstanden, die in der öffentlichen Alltagskommunikation regelhaft vorkommen und somit allgemein sehr verbreitet sind. Sie sind Teil der Gemeinsprache, allgemein verständlich und bezeichnen allseits bekannte Konzepte. In Bezug auf Korpusdaten heißt das, dass diese Ausdrücke über eine hohe Frequenz verfügen. Diese Kriterien erfüllen beispielweise Paare bzw. Gruppen wie halbstündig/ halbstündlich, kindisch/kindlich/kindhaft, lebenslang/lebenslänglich, unehelich/ nichtehelich/außerehelich. Allgemein- und Fachsprache sind in diesem Zusammenhang nicht als getrennte Entitäten zu verstehen. Ausdrücke und Konzepte, die auch häufig außerhalb eines Fachgebietes thematisiert werden und dadurch in der Sprachgemeinschaft regelhaft in öffentlichen Diskussionen vorkommen, können in die Gemeinsprache übergehen. Unterschiedliche Einflüsse, etwa brisante öffentliche Debatten oder Werbung, sorgen dafür, dass sich auch fachsprachliche Paare (z.B. Parodontose/Parodontitis aus der Medizin) bereits der Standardsprache zuordnen lassen. Schwierig ist die Einschätzung darüber, ob frequente Paare wie elektrisch/elektronisch oder effektiv/effizient der öffentlichen Alltagssprache oder dem Fachwortschatz angehören. Der Großteil aller Paronyme ist gemeinsprachlich.

Fachwortschatz wird als ,konstitutives Merkmal der Sprache in Wissenschaft, Technik oder Institutionen“ (Roelcke 2015: 372) betrachtet. Fachsprachliche Kommunikation zeigt besondere Auffälligkeiten in Bezug auf syntaktische 
oder textstilistische Phänomene ebenso wie in Bezug auf ihren Wortschatz (vgl. Roelcke 2010). Fachsprache wird von bestimmten Sprecher^innengruppen genutzt, um einen gezielten, präzisen und schnellen Austausch fachspezifischer Information zu gewährleisten. Fachlexikalische Ausdrücke haben die kommunikative Funktion, bestimmtes fachspezifisches Wissen zu versprachlichen. Sie sind terminologische Bezeichnungen spezifischer relevanter Konzepte und Gegenstände eines Fachgebietes. Das geschieht, damit Eindeutigkeit hergestellt und die Verständlichkeit bei der fachlichen Kommunikation nicht beeinträchtigt wird (siehe Roelcke 2015: 378). Auch Paronyme lassen sich als Bestandteil wissenschaftlicher (z.B. Methode/Methodik/Methodologie) oder technischer (z.B. thermisch/thermal) Fachsprachen sowie als Teil von Verwaltungssprache (z.B. unehelich/nichtehelich/außerehelich) einordnen.

\subsection{Vorkommen und Verteilung}

Auf der Basis von DeReKo wurden 2015 erstmals systematisch korpusgestützt Paronymkandidaten ermittelt und anschließend redaktionell ausgewertet (siehe Schnörch 2015). Das Ergebnis waren 2.010 Paare, die generell als potentielle Paronyme eingestuft wurden, ohne dass genaue Aussagen darüber möglich waren, ob sie im Sprachgebrauch tatsächlich zu sprachlichen Zweifelsfällen führen. In mehreren unabhängigen Abfrageverfahren wurden diese Paare anschließend daraufhin intuitionsbasiert geprüft, ob es sich um Fachwörter im weitesten Sinne handelt. Die größte Schwierigkeit, die sich dabei ergab, war eine Tendenz, Fachwortschatz mit Fremdwortschatz gleichzusetzen. Aber nicht hinter jeder fremdsprachlichen Lexik verbergen sich zugleich Termini einer Fachsprache. Selbst definitorische Erklärungen wie die folgende erleichtern eine klare Zuordnung nur bedingt:

Ein Fachwort (ein Terminus) ist die kleinste bedeutungstragende und syntaktisch frei verwendbare sprachliche Einheit der Kommunikation in einem spezialisierten menschlichen Tätigkeitsbereich. (Roelcke 2015: 373)

Als knifflig wurden solche Paare empfunden, bei denen einer der beiden Ausdrücke unbekannt erschien, der andere aber als allgemeinsprachlich eingestuft wurde (egal/egalitär, Methode/Methodologie, Krise/Krisis). Die Interpretation und Klassifikation eines Paares als ,fachsprachlich' variierte daher stark zwischen 124-534 (im Durchschnitt 329) von 2.010. Dies macht einen durchschnittlichen Anteil von $16 \%$ aus (vgl. Abbildung 1). 


\section{Durchschnittlicher Anteil fachsprachlicher Paronyme}

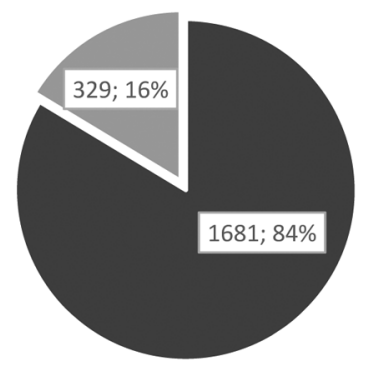

- allgemeinsprachlich achsprachlich

Abbildung 1: Geschätzter Anteil fachsprachlicher Paronyme

Die folgenden Beispiele zeigen einige als Fachwortschatz identifizierte Paare und die ihnen zugeordnete(n) Fachsprache(n):

\begin{tabular}{ll}
\hline Arthritis/Arthrose & Medizin \\
\hline Adaption/Adaptation & Kunst, Soziologie, Biologie, Optik \\
\hline autoritär/autoritativ & Erziehungswissenschaft \\
\hline Egozentrik/Egozentrismus & Psychologie \\
\hline Galaxie/Galaxis & Astronomie, Astrophysik \\
\hline historisch/historistisch/historizistisch & Geschichtswissenschaft \\
\hline Induktion/Induktivität & Elektrotechnik \\
\hline konkav/konvex & Optik \\
\hline Ökonomie/Ökonomik & Wirtschaft \\
\hline oxidativ/oxidiert & Chemie \\
\hline phänomenal/phänomenologisch & Philosophie \\
\hline national/nationalistisch & Politik \\
\hline Stalaktit/Stalagmit & Geologie \\
\hline Synthese/Synthesis & Philosophie \\
\hline temporär/temporal & Sprachwissenschaft, Anatomie \\
\hline thermisch/thermal & Physik \\
\hline virtuell/virtual & Informatik \\
\hline satanisch/satanistisch & Religion \\
\hline
\end{tabular}


Recht stark variiert auch die Vorkommenshäufigkeit der als fachsprachlich markierten Paare in der öffentlichen Gebrauchssprache. Abbildung 2 zeigt ein Beispiel einer Auswertung der nach sechs Frequenzschichten verteilten fachsprachlichen Paronyme. ${ }^{6}$ In diesem Beispiel wurden 324 Fachsprachenpaare (aus der Gesamtmenge von 2.010 Paronympaaren) identifiziert. Es enthält damit eine durchschnittlich hohe Anzahl und steht exemplarisch für vergleichbare Auswertungen, weil die Verteilungsverhältnisse jeweils recht ähnlich sind.

\section{Vorkommen fachsprachlicher Paronyme}

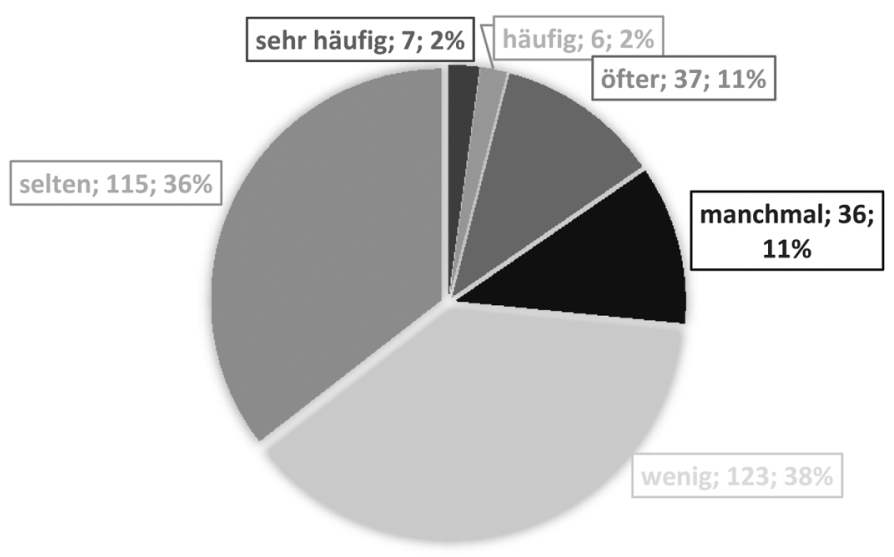

Abbildung 2: Verteilung fachsprachlicher Paronyme nach sechs Frequenzschichten

Die Frequenzschicht ,sehr häufig‘ enthält 2\% aller fachsprachlichen Paronyme, ebenso sind $2 \%$,häufig' belegt, $11 \%$,öfter vorkommend. Der Großteil der als fachsprachlich eingeschätzten Paronyme kommt in der öffentlichen Kommunikation ,wenig' ( $38 \%$ ) bis ,selten' ( $36 \%$ ) vor. Unter den selten belegten Ausdrücken sind zahlreiche Wortbildungsprodukte, etwa Komposita (z.B. Wasserstofftechnik/ Wasserstofftechnologie) oder Negationen (z.B. denaturieren/denaturalisieren).

\footnotetext{
6 Die ermittelten Frequenzgruppen orientieren sich am Vorkommen im öffentlichen Sprachgebrauch (DeReKo), nicht in der Fachsprache selbst. Die Frequenzschichten wurden in Anlehnung an die DeReKo-Häufigkeitsklassen (vgl. dazu Müller 2015: 142) festgelegt. Häufigkeitsklasse 8-11 = sehr häufig (mind. ein Paronym des Paares), 12-15 = häufig (beide Paronyme), 12-15 = öfter (mind. ein Paronym des Paares), 16-19 = manchmal (beide Paronyme), 16-19 = wenig (mind. ein Paronym des Paares), 20-29 = selten (mind. ein Paronym des Paares).
} 
Derartige Auswertungen sollten nur als grobe Einschätzungen gesehen werden. Das liegt auch daran, dass u.a. Ausdrücke wie unehelich/nichtehelich/außerehelich oftmals nicht als Fachwortschatz eingeordnet werden, obwohl sie eine bevorzugte Verwendung in der Verwaltungssprache aufweisen. Ebenso wurden lebenslang/lebenslänglich oder glaubhaft/glaubwürdig nicht als rechtswissenschaftliche Ausdrücke zur Charakterisierung von Urteilen bzw. der Überzeugungskraft von Aussagen oder Zeugen erkannt. In beiden Fällen verfügen diese Paare/ Gruppen auch über allgemeinsprachliche Verwendungen, sind in lexikalischer bzw. morphologischer Hinsicht nicht fremdsprachlich und werden daher als alltagssprachlich eingestuft. Auf der anderen Seite gehören viele eindeutige Fachausdrücke mittlerweile zum allgemeinen Wortschatz, z.B. Parodontose/Parodontitis, und spielen auch außerhalb bestimmter Berufsgruppen und deren Fachgebieten (hier Medizin) eine größere Rolle. Dieses Phänomen beobachtet auch Roelcke (2015: 383) in Bezug auf die Bewertung von Termini, die polysem sind und neben einer fachsprachlichen Lesart mehrere nichtfachsprachliche Verwendungen aufweisen, z.B. Wurzel (Zahnmedizin/Linguistik/Mathematik). Die kontextgebundene Verwendung spielt folglich bei der Bewertung eine zentrale Rolle. Die Auswertungen erfolgten aber lexembezogen und nicht im Zusammenhang mit einer semantischen Analyse. Daher ist wohl der Anteil ,häufiger‘ oder ,sehr häufiger‘ Paronyme mit fachsprachlicher Verwendung höher anzusetzen. Unter den ,sehr häufigen“ bis ,öfter` gebrauchten Ausdrücken sind zahlreiche Paare, die auch Überschneidungen zu bildungssprachlichen Paronymen aufweisen (z.B. effizient/effektiv, sensibel/sensitiv, rational/rationell, formell/formal) und die aufgrund ihres fremdsprachlichen Charakters mehrheitlich als fachsprachlich eingeordnet wurden (siehe dazu Kapitel 5). Handelt es sich z. B. bei effektiv um ein Fachwort, wenn damit eine Therapie oder eine Behandlung charakterisiert wird und der Ausdruck überwiegend in medizinischen Kontexten vorkommt? Ist sensibel ein Fachwort der Ökologie, wenn es im Zusammenhang mit Ökosystemen, Gebieten und Zonen, Naturraum vorkommt? Die Abgrenzungen zur thematischen Gebundenheit bleiben gleichfalls unscharf. So kann oft erst nach einer gründlichen korpussemantischen Untersuchung genauer gesagt werden, ob es diskursive Präferenzen oder fachsprachliche Verwendungen für ein bestimmtes Paar gibt.

\subsection{Dynamik in der Verwendung}

Alltagssprachliche Paronyme weisen ein größeres Potenzial auf verwechselt zu werden als rein fachsprachliche Paronyme, die stärker fachspezifischen, definitorischen Restriktionen unterliegen. Fachsprachliche Paronyme, die in der öffentlichen Gebrauchssprache als geläufig einzustufen sind oder allgemeinsprachliche 
Paronyme, die zusätzlich eine fachsprachliche Verwendung aufweisen, verhalten sich teilweise anders als solche, die ausschließlich in einem Register/einer Varietät vorkommen. Die Ursachen für diese semantische Dynamik sind vielfältig. So hängt es u.a. davon ab, ob beide Ausdrücke eines Paares als fachsprachlich markiert sind, oder es ob zu Verwechslungen kommt, weil ein fachsprachlicher Ausdruck mit einem allgemeinsprachlichen Ausdruck lautlich, inhaltlich oder ausdrucksseitig ähnlich ist. Unsicherheiten entstehen auch, wenn es sich um morphologisch durchsichtige Ausdrücke handelt, die semantisch in der Alltagssprache andere Konzepte bezeichnen, oder wenn die beiden Ausdrücke eines paronymen Paares in zwei verschiedenen Fachsprachen anzutreffen sind. Solche Diskrepanzen zwischen allgemeiner Gebrauchssprache und fachsprachlichen Verwendungen sind bei Paronymen öfter zu beobachten. Nachfolgend sollen vier sehr unterschiedliche Beispiele näher betrachtet werden: Parodontose/Parodontitis, lebenslang/lebenslänglich, unehelich/nichtehelich/außerehelich sowie Methode/Methodologie/Methodik. Diese verdeutlichen semantische Divergenzen zwischen Alltagsgebrauch und medizinischem bzw. juristischem Gebrauch, zwischen öffentlicher Gebrauchs- und Verwaltungssprache sowie Verschiebungen in der allgemeinen Wissenschaftskommunikation.

\subsubsection{Medizin: Parodontitis/Parodontose}

In der Medizin stehen Termini mit der Endung -itis für entzündliche Erkrankungen und solche mit der Endung -ose für atrophische Erkrankungen, d.h. solche, die für die Rückbildung von Gewebe stehen. Daher werden solche Ausdrücke voneinander unterschieden und abgegrenzt. Die entzündliche Erkrankung des gesamten Zahnhalteapparates wird als Parodontitis bezeichnet. Der nicht-entzündliche Rückgang des Zahnfleisches und die damit verbundene Freilegung des Zahnhalses bezeichnet man als Parodontose. In Fachlexika werden beide Bezeichnungen klar getrennt:

Parodontitis: entzündliche Zerstörung des Zahnhalteapparates (Parodont). Führt ohne Behandlung zu Zahnlockerung und schließlich zum Verlust des betroffenen Zahnes. Die Parodontitis geht einher mit dem Auftreten bestimmter Bakterien. (http://www.gesundheit.de/ lexika/zahn-lexikon/parodontitis.)

Parodontose: degenerative Form der Parodontopathie mit Schwund des marginalen Zahnhalteapparates aufgrund primär-regressiver, nicht entzündl. Prozesse; mit rel. geringer Taschenbildung, aber Zahnlockerung u. später -verlust. [...] (http://www.gesundheit.de/lexi$\mathrm{ka}$ /medizin-lexikon/parodontose.) 
Die Bezeichnung einer Parodontitis als Parodontose wäre daher fachlich nicht korrekt. In der allgemeinen Gebrauchssprache werden Parodontitis und Parodontose hingegen synonym verwendet. Man bezieht sich mit beiden Ausdrücken auf eine nicht näher differenzierte Erkrankung des Zahnhalteapparates. Der Ausdruck Parodontose wird dann aus fachsprachlicher Sicht als wissenschaftlich falsch bewertet. Das zeigen u.a. Konstruktionen wie ... die Parodontose (wissenschaftlich korrekt: Parodontitis), ... Parodontitis, fälschlich oft Parodontose genannt, ... Parodontose oder wissenschaftlich korrekt Parodontitis genannt.

1. Jeder kann sein Schicksal in die Hand nehmen - morgens, mittags und abends. Mit Hilfe von speziellen Zahnbürsten und Zahnseide. Denn hygienische Missstände in der Mundhöhle können die Lebenserwartung verkürzen. Nicht Karies ist hier die Gefahr, sondern die Parodontose (wissenschaftlich korrekt: Parodontitis). (Stern, 06.05.2004, Risiko Entzündung)

In der Alltagskommunikation verwendet man Parodontose regelhaft anstelle von Parodontitis. Im Gegensatz zur fachlichen Bewertung ,inkorrekt‘ (s.o.) wird hier allerdings nur eine Synonymie (nicht wertend) kommentiert, möglicherweise sogar manifestiert. Diese landläufige Gleichsetzung wird auch oft metasprachlich markiert:

.... Parodontitis, im Volksmund auch/oft „Parodontose“ genannt, ...

.... Parodontitis, in der Umgangssprache auch „Parodontose“ genannt, ...

.... Parodontitis (umgangssprachlich Parodontose genannt), ...

.... Parodontitis (umgangssprachlich bekannt als Parodontose), ...

.... Parodontitis (landläufig Parodontose genannt), ...

Die Alltagssprache unterscheidet oftmals nicht zwischen einer Entzündung des Zahnfleisches oder einer Erkrankung des Zahnhalteapparates mit Gewebeschwund. Stattdessen werden beide Ausdrücke für sämtliche Erkrankungen und Entzündungen des Zahnbettes, -fleisches oder -halteapparates genutzt. So werden beide als medizinischer Terminus für eine Zahnfleischentzündung genutzt, siehe dazu die Synonymbelege 2 und 3.

2. Chronische, bakterielle Entzündungen wie eine Parodontitis (Zahnfleischentzündung) können nicht nur Zahnverluste zur Folge haben. (Rheinische Post, 29.05.2009, Gesundheit beginnt im Mund.)

3. Die weitreichenden Folgen von Parodontose werden nach Experten-Meinung in Deutschland unterschätzt. „Die Zahnfleisch-Entzündung erhöht das Risiko für Herzinfarkte, Schlaganfälle und Frühgeburten“, sagte der Präsident der Deutschen Parodontose-Hilfe [...]. (Mannheimer Morgen, 30.04.1999, Parodontose wird unterschätzt.) 
Speziell in Kontexten mit Thematisierungen der Allgemeingesundheit wird nicht zwischen den beiden Ausdrücken unterschieden. Das kontextuelle Umfeld der Ausdrücke weist infolgedessen zahlreiche Gemeinsamkeiten auf: Beide werden häufig mit Ausdrücken wie Zähne, Zahnarzt, Hauptursache, Ursachen und Patient assoziiert. Sowohl Parodontitis als auch Parodontose sind Entzündungen, die behandelt oder denen vorgebeugt werden sollten.

Eine Ursache für unterschiedliche Verwendungen in der Allgemeinsprache und in der Fachsprache könnte am Gebrauch beider Ausdrücke in der Werbesprache zum Bewerben von Zahnpasta liegen. So erklärt z.B. das Gesundheitsportal medhost die Verwechslung wie folgt:

Die Parodontitis - im Vergleich zur Parodontose - ist ein entzündlicher Krankheitsprozess, der zum Verlust des Zahnhalteapparates führt. Die Begriffsverwirrung hat ihren Ursprung in der TV-Zahnpasta-Werbung. Hier wurde der Begriff „Parodontose“ für die marginale (siehe unten) Parodontitis verwendet. (https://www.medhost.de/gesundheit-lexikon/parodon tose.html)

Den Hinweis auf einen ursächlichen Einfluss der Werbung findet man auch in einigen Korpusbelegen.

4. Bakterien und Zahnbeläge, mangelnde Mundhygiene, aber auch Veranlagung oder Erkrankungen wie Diabetes können zur so genannten Parodontitis, aus der ZahnpastaWerbung als Parodontose bekannt, führen. (Saarbrücker Zeitung, 12.07.2001, Der Parodontose den Zahn ziehen.)

Die seitens der Werbung initiierte Verwendung von Parodontose im Sinne von Parodontitis hat eine kontinuierliche Rezeption zur Folge und fand so in der Allgemeinsprache rasch Verbreitung. Das führte zu einer Annäherung und Gleichsetzung. Bei anderen Wortpaaren des Musters -itis/-ose (Arthritis/Arthrose, Dermatitis/Dermatose, Gastritis/Gastrose, Neuritis/Neurose, Spondylitis/Spondylose) haben solche Semantisierungsprozesse nicht stattgefunden. So werden z.B. Arthritis/Arthrose oder Neuritis/Neurose in der Alltagssprache genauso differenziert verwendet wie in der Fachsprache, es erfolgte keine Synonymisierung.

\subsubsection{Rechtswissenschaft: lebenslang/lebenslänglich}

Lebenslang und lebenslänglich sind in der öffentlichen Gebrauchssprache bedeutungsgleich. Beide Ausdrücke kommen in allgemeinen Diskursen und Themenbereichen vor, aber auch in juristischen Kontexten wie in Berichten zu Urteilsverkündungen mit dem geringen Unterschied, dass lebenslang weitaus 
häufiger auftritt als lebenslänglich. Beide lassen sich gleichermaßen nach drei Verwendungsmustern ausdifferenzieren. Auch die Bedeutungsgleichheit lässt sich in allen drei Fällen anhand von Synonymbelegen (Belege 5-7) bestätigen:

1) ,bis zum Tod andauernd': charakterisiert einen Sachverhalt oder eine Handlung als bis zum Tod der davon betroffenen Person andauernd (z.B. Lernen, Sperre, Wohnrecht, Freundschaft, Rente, Garantie)

5. Johnson, der nach seinem 100-m-Sieg 1988 bei den Olympischen Spielen in Seoul der Einnahme von Anabolika überführt worden war und fünf Jahre später bei einem Wettkampf in Montreal erneut erwischt wurde, war 1993 vom Internationalen Leichtathletik-Verband (IAAF) lebenslang gesperrt worden. Das Council des IAAF hatte nach Johnsons Einspruch erst im Sommer dieses Jahres vor der Weltmeisterschaft in Sevilla die lebenslängliche Sperre bestätigt. (Nürnberger Nachrichten, 17.11.1999, S. 30.)

2) ,die maximale Freiheitsstrafe betreffend': charakterisiert im Recht eine Freiheitsstrafe als die höchstmögliche, maximale Strafe, die ein juristisches System definiert (z.B. Haft, Haftstrafe, Strafe, Freiheitsstrafe, Verurteilung)

6. Das Gericht bestätigte das von einem Geschworenengericht vor einem Jahr gefällte Urteil gegen die 33 Jahre alte Waltraud Wagner, die als die zentrale Figur des mörderischen Quartetts gilt, wegen 15-fachen Mordes, 17-fachen Mordversuchs und zwei Fällen von schwerer Körperverletzung zu einer lebenslangen Freiheitsstrafe. Ebenfalls bestätigt wurde das lebenslängliche Strafmaß für die 30jährige Irene Leidolf, der fünf vollendete und zwei versuchte Morde angelastet wurden, und die 20-jährige Haftstrafe für die 52-jährige Stefanija Mayer wegen siebenfachen versuchten Mordes und einer fahrlässigen Tötung. (Süddeutsche Zeitung, 16.05.1992.)

3) ,Urteil‘: bezeichnet das Gerichtsurteil, das im Rahmen eines juristischen Systems die maximale Freiheitsstrafe darstellt

7. In diesem Fall würde vermutlich lebenslänglich verhängt, zumindest für den 20-jährigen Serkan A. Allerdings nur in der Theorie, denn in Amerika würde niemand mit 40 Vorstrafen frei herumlaufen. In einigen Staaten, wie Kalifornien, kann bei der dritten Tat lebenslang ausgesprochen werden. (Die Zeit, 10.07.2008.)

Es gibt allerdings einen entscheidenden Unterschied zwischen dem Gebrauch der beiden Adjektive in der Allgemeinsprache und in der juristischen Fachsprache. Lebenslang und lebenslänglich werden in juristischen Kontexten des deutschen Strafrechts abweichend vom Alltagsverständnis gebraucht.

Dennoch gilt, dass bei einem Urteil, das mit lebenslänglich bezeichnet wird bzw. mit einer lebenslangen Freiheitsstrafe (etwa bei Mord nach Deutschem Strafgesetzbuch), der Verurteilte die Möglichkeit hat, nach Verbüßung von 15 Jahren 
Haft die Aussetzung der Strafe zu beantragen. ${ }^{7}$ Einen lebenslangen Freiheitsentzug, also tatsächlich bis zum Ende des Lebens, kann ein Verurteilter nur dadurch erfahren, dass nach der formalen Freiheitsstrafe die Sicherungsverwahrung hinzukommt. Lebenslänglich und lebenslang beziehen sich also in Deutschland auf den Freiheitsentzug auf unbestimmte Zeit, mindestens aber auf 15 Jahre. Dass die Alltagsvorstellung von lebenslanger Haft im Sinne einer Haftstrafe bis zum Tod existiert, wird anhand zahlreicher Klarstellungen deutlich (vgl. Beleg 8).

8. „Lebenslänglich“ sind rund 20 Jahre. Justizminister Banzer betont Resozialisierungsgebot auch bei Höchststrafe. Strafvollzug in Hessen: „Lebenslang“ bedeutet im hessischen Strafvollzug keineswegs Haft bis zum Tode. (Frankfurter Rundschau, 23.10.2006, S. 15.)

Mit lebenslang oder lebenslänglich wird nicht die Strafverbüßung bis zum Tode bezeichnet. Die Annahme, dass ein Strafmaß ,lebenslang‘ bis zum Tod andauert, besteht z.T. deshalb, weil die Ausdrücke einerseits semantisch durchsichtig scheinen und damit wörtlich verstanden werden, und andererseits diese Bedeutung in Verwendungen wie lebenslanges Lernen/Wohnrecht oder lebenslang/lebenslänglich an etwas leiden sich tatsächlich auf das Konzept ,ein Leben lang، beziehen. Urteile wie zweimal lebenslang/lebenslänglich sind in Deutschland nicht zulässig, da nach deutschem Recht Strafen nicht addiert werden. Solche Urteile beziehen sich auf Rechtsprechungen anderer Länder, wie z.B. den USA. Dort bedeutet lebenslang/lebenslänglich tatsächlich bis zum Tode des Verurteilten andauernd. Beide Ausdrücke müssen hinsichtlich ihrer juristischen Bedeutung nach der Rechtsprechung eines jeden einzelnen Staates definiert werden. Im Strafgesetzbuch (§ 38 Abs. 1) selbst gibt es den gebräuchlichen Ausdruck lebenslänglich nicht. Dort kommt nur die „lebenslange Freiheitsstrafe“ vor. Bei diesem Wortpaar gibt es also sowohl Unterschiede bezüglich der Geläufigkeit beider Ausdrücke in der Alltagskommunikation und in juristischen Fachtexten als auch irrtümliche Annahmen über die fachspezifische Bedeutung der Ausdrücke in der Alltagssprache.

\subsubsection{Verwaltungssprache: unehelich/nichtehelich/außerehelich}

Die Verwaltungssprache kennzeichnet ein eher förmlicher, rechtlich-institutioneller Sprachgebrauch, der innerhalb und außerhalb von Organisationen, Behörden, Verwaltungsstellen sowie Ämtern vorherrscht. Die Ausdrucksweise

7 Siehe https://www.gesetze-im-internet.de/stgb/_211.html. 
(meist des Schriftverkehrs) ist distanziert, unpersönlich bis neutral. Geprägt ist diese durch einen komprimiert abstrakten Stil, der dennoch den Anspruch auf inhaltliche Vollständigkeit und Eindeutigkeit bei der Kommunikation besitzt und gleichzeitig gesetzliche Vorgaben in Bezug auf kommunikative Anforderungen zur Wahrung von Genauigkeit und Neutralität umsetzt. Um diese Neutralität gewährleisten zu können, werden bei sich verändernden gesellschaftlichen Bedingungen soziokulturelle oder politische Diskurse bewusst gesteuert. Man denke etwa an Political Correctness. Im Rahmen dieses Konzepts ist man bemüht, stigmatisierte durch semantisch unbesetzte Ausdrücke zu ersetzen, vor allem solche, die sich auf Bezeichnungen oder Charakterisierungen von Personen beziehen. Um einen solchen Fall handelt es sich bei der Einführung des Ausdrucks nichtehelich, der dem Adjektivpaar unehelich/außerehelich einen dritten Ausdruck zur Seite stellt. 1970 wurde mit dem neuen Nichtehelichenrecht (siehe dazu Berg/Sonnenfeld 2012) der Ausdruck unehelich offiziell durch nichtehelich ersetzt.

Mit dem Inkrafttreten des Gesetzes über die rechtliche Stellung der nichtehelichen Kinder am 01.07.1970 wurden die unehelichen Kinder nichtehelich genannt. (Gesetz über die rechtliche Stellung der nichtehelichen Kinder vom 19.08.1969 [BGBl. I 1243])

Neben der juristischen Gleichstellung, die mit dem Gesetz angestrebt wurde, sollte durch die Wahl eines neutralen, konnotativ unbesetzten Ausdrucks auch die sprachliche Stigmatisierung von unehelich beseitigt werden.

9. Justiz und Politik wollen die Benachteiligung unverheirateter Frauen beheben, die sich nach der Trennung um die Kinder kümmern. Es ist noch gar nicht so lange her, da stand im Bürgerlichen Gesetzbuch kurz und knapp: „Ein uneheliches Kind und dessen Vater gelten als nicht verwandt.“ Erst am 1. Juli 1970 fiel diese extreme Diskriminierung weg. Seit dieser Reform, die übrigens auch aus dem „unehelich“ ein „nichtehelich“ machte, ist im Dreieck nichteheliches Kind - Mutter - Vater viel geschehen. (Süddeutsche Zeitung, 06.07.2006, S. 2.)

In der offiziellen Verwaltungssprache hat sich der Ausdruck nichtehelich zur näheren Charakterisierung von Kindern nicht miteinander verheirateter Eltern bis 1998 durchgesetzt. In Verwaltungstexten wird daher nur ein Gegensatz zwischen ehelich und nichtehelich thematisiert. Der Ausdruck außerehelich spielt in der Fachsprache keine Rolle. Mit der Kindschaftsreform von 1998 wird die juristische Gleichstellung ehelicher und nichtehelicher Kinder angestrebt und die Benachteiligung nichtehelicher Kinder aufgehoben. Der Untertitel des Gesetzes ${ }^{8}$ macht die

8 Unterhaltspflicht im Bürgerlichen Gesetzbuch, §§ 1615a-1615n BGB. 
Abschaffung des Ausdruckes nichtehelich kenntlich. Es trägt seit 1. Juli 1998 die Überschrift „Besondere Vorschriften für das Kind und seine nicht miteinander verheirateten Eltern“. Es heißt nun zur Vermeidung der Begriffe unehelich und nicht ehelich im BGB u.a. in § 1791c Abs. 1: Mit der Geburt eines Kindes, „dessen Eltern nicht miteinander verheiratet sind [...]“. Das deutet darauf hin, dass der gesteuerte lexikalische Ersatz von unehelich mit nichtehelich im Jahre 1970 nicht zur gewünschten Aufhebung der Stigmatisierung geführt hat.

In der öffentlichen Gebrauchssprache verhalten sich die drei Ausdrücke wie folgt: Alle drei Ausdrücke unehelich, nichtehelich und außerehelich kommen regelhaft vor. Der frequenteste Ausdruck ist unehelich, also genau dasjenige Adjektiv, das in der Verwaltungssprache ersetzt wurde. Hier zeigt sich demnach eine große Diskrepanz in der Vorkommenshäufigkeit des Ausdrucks in den beiden Varietäten. Unehelich, nichtehelich und außerehelich können in Bezug auf Verwendungen mit einzelnen Personen und Sachverhalten bedeutungsgleich verwendet werden. Sie kommen mit identischen Kollokatoren vor (z.B. Kind, Sohn, Tochter). Unehelich und nichtehelich weisen zusätzlich Gemeinsamkeiten in Verwendungen auf, in denen Sachverhalte wie Lebensformen und Partnerschaften näher charakterisiert werden. Die beiden Adjektive bezeichnen diese als ,keine amtlich zertifizierte Ehe aufweisend'. Nichtehelich und außerehelich besitzen jeweils noch eine weitere Verwendung. Personen, die als nichtehelich bezeichnet werden, wie z.B. Eltern oder Paare, werden als unverheiratet charakterisiert, können jedoch nicht als unehelich oder außerehelich bezeichnet werden. Außerehelich kann sich aber auch überwiegend auf Sachverhalte (und Prozesse) beziehen, die neben einer bestehenden Ehebeziehung ablaufen, z.B. Affäre, Beziehung. Diese Sachverhalte (oder Handlungen) existieren oder erfolgen nicht mit unverheirateten Personen. Darin liegt der Unterschied zu nichtehelich, sie betreffen Personen, die durchaus in einer Ehe leben (zumindest einer von ihnen). Tabelle 1 stellt die möglichen Verwendungen zusammenfassend dar.

Tabelle 1: unehelich/nichtehelich/außerehelich im Vergleich

\begin{tabular}{lll}
\hline \multicolumn{1}{c}{ unehelich } & \multicolumn{1}{c}{ nichtehelich } & \multicolumn{1}{c}{ außerehelich } \\
\hline & \multicolumn{1}{c}{,außerhalb einer Ehe } \\
\hline bezeichnet eine Person (z.B. & bezeichnet eine Person (z.B. & bezeichnet eine Person (z.B. \\
Kind, Sohn, Tochter, Mütter, & Kinder, Väter, Mütter, Babys, & Kind, Tochter, Sohn) als \\
Väter, Neugeborene, & Sohn, Tochter, Nachwuchs) als & außerhalb der Ehe existent \\
Sprössling, Baby, & außerhalb der Ehe existent \\
Nachkommen) als außerhalb & & \\
der Ehe existent & & \\
&
\end{tabular}


Tabelle 1: (fortgesetzt)

\begin{tabular}{|c|c|c|}
\hline unehelich & nichtehelich & außerehelich \\
\hline $\begin{array}{l}\text { bezeichnet ein Ereignis } \\
\text { (Geburt, Herkunft, } \\
\text { Schwangerschaft) als } \\
\text { außerhalb der Ehe existent }\end{array}$ & $\begin{array}{l}\text { bezeichnet ein Ereignis (z.B. } \\
\text { Geburt) als außerhalb der Ehe } \\
\text { existent }\end{array}$ & $\begin{array}{l}\text { bezeichnet ein Ereignis (z.B. } \\
\text { Geburt, Schwangerschaft) als } \\
\text { außerhalb der Ehe existent }\end{array}$ \\
\hline \multicolumn{3}{|c|}{ „keine Ehe aufweisend“ } \\
\hline $\begin{array}{l}\text { weist einer menschlichen } \\
\text { Beziehung den Status zu, keine } \\
\text { Ehe aufzuweisen (z.B. } \\
\text { Beziehung, Lebensge- } \\
\text { meinschaft, Verhältnisse, } \\
\text { Partnerschaften) }\end{array}$ & $\begin{array}{l}\text { weist einer menschlichen } \\
\text { Beziehung den Status zu, keine } \\
\text { Ehe aufzuweisen (z.B. } \\
\text { Lebensgemeinschaften, Part- } \\
\text { nerschaften, Gemeinschaften, } \\
\text { Beziehungen, Lebensverhält- } \\
\text { nisse) }\end{array}$ & \\
\hline \multicolumn{3}{|c|}{,nicht verheiratet' } \\
\hline & $\begin{array}{l}\text { weist Personen den Status zu, } \\
\text { nicht verheiratet zu sein (z.B. } \\
\text { Lebenspartner, Paare, Partner, } \\
\text { Eltern) }\end{array}$ & \\
\hline & & ,neben einer existierenden Ehe \\
\hline & & $\begin{array}{l}\text { bezeichnet einen Sachverhalt } \\
\text { oder einen Prozess dahinge- } \\
\text { hend, neben einer existieren- } \\
\text { den Ehe abzulaufen (z.B. } \\
\text { Affäre, Beziehung, Sex, Ge- } \\
\text { schlechtsverkehr, Verhältnis) }\end{array}$ \\
\hline
\end{tabular}

Besonders in der Verwendung von nichtehelich ,nicht verheiratet ${ }^{\star}$ kommt die verwaltungssprachliche Bedeutung zum Ausdruck. Ansonsten sind unehelich und nichtehelich bedeutungsgleich, mit dem einen Unterschied, dass die zweite Verwendung von nichtehelich (,keine Ehe aufweisend') die dominanteste Bedeutung ist, während bei unehelich die 1. Verwendung (,außerhalb einer Ehe`) am gebräuchlichsten ist. Wichtig bei der Verwendung der Adjektive sind auch Bewertungen, die erzeugt werden. Im Zusammenhang mit Kontexten ,außerhalb einer Ehe', die mit allen drei Adjektiven ausgedrückt werden können, besteht die Tendenz, die Adjektive nichtehelich/außerehelich als neutral bewertete Ausdrücke für Personen zu nutzen, die außerhalb einer Ehe hervorgegangen sind oder leben. Unehelich wird dagegen stärker, wenn auch nicht ausschließlich, in negativ bewerteten Kontexten genutzt. Dies geschieht vor allem in historischen Texten oder in Texten, in denen über vergangene Zeiten berichtet wird. Auf diese Weise werden dann Personen 
charakterisiert, die außerhalb einer anderseits bestehenden Ehe hervorgegangen sind oder leben, als dies zugleich in sozialer oder religiöser Hinsicht als Stigma bzw. veraltet auch als illegitim galt. In derartigen Kontexten tauchen zudem öfter negativ besetzte Ausdrücke auf, z.B.: Affäre, Schmach, Sünde.

10. Der Gegenkandidat Hubers für den Parteivorsitz, der Frauenverbraucherminister Horst Seehofer, hat seine Chancen krass gemindert, weil er sich eine Geliebte und unehelichen Nachwuchs zugelegt hat. (die tageszeitung, 20.07.2007, S. 5, Die Stoiber-Bilanz.)

11. Ich bin unehelich, deshalb darf ich nicht ministrieren. Im Amt muss ich an der Tür stehen bleiben, damit ich nicht hören kann, wenn meine Mutter flüstert, der Junge ist unehelich. Unehelich ist eine Schande. (FOCUS, 22.12.2006, S. 84.)

Zusammenfassend lässt sich festhalten, dass es Unterschiede in der allgemeinen Gebrauchssprache und in der Verwaltungssprache bei der Verwendung von Ausdrücken gibt, die das Konzept Nicht verheiRatet bezeichnen. Durch gesellschaftliche Veränderungen, juristisches Eingreifen und sprachliche Steuerung konnte die Stigmatisierung des Konzeptes nicht verheiratet verblassen. Dadurch wurden semantische Wandelprozesse in Gang gesetzt. Diese neutralisieren nach und nach unehelich als negativ besetzten Ausdruck; sie etablieren ein neu abgeleitetes Adjektiv nichtehelich im Alltagsgebrauch, wodurch unehelich sukzessive ersetzt wird. Eine Folgeentwicklung ist allerdings, dass nichtehelich bald auf eine Weise gebraucht wird, die unehelich und außerehelich nicht aufweist: Nur nichtehelich kann mit Bezug auf unverheiratete Personen oder auf deren Status verweisen.

So wie sich der Begriff für die elterliche Sorge wandelte, so änderte sich auch der für das Kind, welches nicht in einer Ehe geboren wurde von unehelich über nichtehelich bis zu dem „Kind, dessen Eltern nicht miteinander verheiratet sind“. (Berg \& Sonnenfeld 2012: 2)

Der Eingriff über die sprachliche Steuerung der Verwaltungssprache hat zwar das sprachliche Bewusstsein verändert, aber nicht zum Wegfall von unehelich im Alltag geführt. Die bedeutungsgleichen Verwendungen sind dabei keineswegs die häufigsten Kontexte. Und so sind eigene typische, bevorzugte Gebrauchsvarianten zu erkennen, die zeigen, dass die Ausdrücke beginnen, sich in der Alltagssprache voneinander zu unterscheiden. Ähnliche Beobachtungen machen Mell, Schnörch und Storjohann (2019: 65-66) auch bei den Ausdrücken antisozial/asozial/unsozial/dissozial.

Eine solche ,künstliche‘ Einführung neuer Wörter, die sich lediglich etwa durch die Wahl der Vorsilbe unterscheiden, wird häufig in der Amtssprache oder in Fachsprachen gebildet. Von dort aus diffundieren sie dann nach und nach in die Allgemeinsprache, in der es dann zu sprachlicher Rivalität kommt. Auf der Ebene der Diskurse haben wir es dann mit Spezialdis- 
kursen oder auch Fachtermini zu tun, denen die Paronyme zuzuordnen sind und die sich mit dem Diskurs des öffentlichen Sprachgebrauchs ähnliche Konzepte teilen.

Die Wandelprozesse sind möglicherweise noch nicht abgeschlossen. Drei miteinander konkurrierende Ausdrucke beeinflussen sich gegenseitig. Und so bleibt abzuwarten, in Richtung welcher Beziehung sich diese drei Paronyme entwickeln.

\subsubsection{Allgemeinwissenschaft: Methode/Methodologie/Methodik}

Unter den leicht verwechselbaren Ausdrücken befinden sich auch Paronyme, die allgemein wissenschaftssprachlicher Natur sind, da sie überwiegend in akademischen Kontexten vorkommen (z.B. empirisch/empiristisch, Synthese/Synthesis, These/Thesis, Evaluation/Evaluierung, Konzept/Konzeption, konzeptionell/konzeptuell, methodisch/methodologisch, Methode/Methodologie/Methodik). Diese Gruppe ist sehr vielfältig, zahlreiche Paare sind keiner speziellen Fachsprache zuzuordnen. Manchmal weist nur ein Bestandteil eines Paares einen fachsprachlichen Bezug auf. In anderen Fällen verfügt ein Paronym sowohl über eine allgemeine als auch eine fachsprachliche Verwendung. ${ }^{9}$ Besonders interessant sind solche Paronyme, die der alltäglichen, fächerübergreifenden Wissenschaftssprache (Ehlich 2000) zuzuschreiben sind, also solche wie Methode/Methodik/ Methodologie. Sie sind einerseits typisch für die allgemeine Gebrauchssprache und verfügen über eine hohe Frequenz in allgemeinsprachlichen Korpora. Andererseits besitzen sie auch in der wissenschaftssprachlichen Kommunikation besondere Funktionen der wissenschaftlich-terminologischen Abgrenzung und gehören zu jenem Inventar, das dem wissenschaftlichen Austausch dient. Ihre gleichzeitige Verwendung sowohl in der alltagssprachlichen als auch in der wissenschaftlichen Kommunikation kann zur Etablierung von semantischen Nuancen führen, die dann Auswirkungen auf Bedeutungsentwicklungen haben und zu größerer Variabilität im Gebrauch führen. Das trifft vor allem auf Methode zu. Diese Variabilität kann auch sprachliche Unsicherheiten oder gar fehlerhaften Gebrauch zur Folge haben.

9 Synthese etwa hat eine fachsprachliche Verwendung in philosophischen Kontexten, wird häufig im Zusammenhang mit Kant und Hegel erwähnt und bezieht sich dort auf eine Einheit aus verschiedenen, oft gegensätzlichen Vorstellungen, Theorien oder Ansätzen. Gleichermaßen kann man mit Synthese auch allgemeinere Bedeutungen ausdrücken. Wird Synthese im allgemeinen Sinn als ,Mischung“ oder ,Einheit‘ gebraucht, handelt es sich weniger um eine allgemeine, bildungssprachliche Verwendung. 
Das Triplett Methode/Methodik/Methodologie zeigt eine Tendenz zur Synonymisierung in der öffentlichen Alltagssprache, was umgekehrt zu einer Tendenz der ungenügenden Präzisierung in der Wissenschaftssprache führt. In der Datengrundlage wird deutlich, dass sich alle drei Nomen generell auf zwei Kontexte beziehen. Zum einen bezeichnen sie eine fachliche Verfahrens- oder Arbeitsweise, die bestimmten Regeln und Abläufen unterliegt und gezielt zur Umsetzung bestimmter Pläne bzw. zur Durchführung bestimmter Analysen oder Experimente genutzt wird. Es ist nicht immer leicht, davon Kontexte abzugrenzen, in denen sich Methode und Methodik auf allgemeine Vorgehens- und Herangehensweisen im Sinne einer Strategie beziehen. Sie bezeichnen somit eine auf die Erreichung eines bestimmten Ziels hin ausgerichtete, planmäßige Praktik. Damit erfährt insbesondere Methodik eine Bedeutungserweiterung und eine Annäherung an den allgemeinsprachlichen Ausdruck Methode in der öffentlichen Gebrauchssprache. Semantische Unterschiede zwischen den drei Ausdrücken sind hier schwer auszumachen (siehe Beispiele 15 und 16).

15. Die Biotechnologie ist „eine universell einsetzbare Methode“, beschreibt Riedel das Verbindende der bisher „relativ unabhängigen Sparten Gesundheit und Ernährung“. Da die Erbsubstanz (DNA) in allen Organismen einheitlich sei, könne die Genomforschung „mit der gleichen Methodik kranke oder infizierte Zellen bei Pflanzen, Tieren oder Menschen untersuchen“. (Frankfurter Rundschau, 06.06.1998, S. 13.)

16. Mit der Veränderung der Grenzwerte erzielt man dann viel günstigere Zahlen bei der Armutsmessung. Ähnlich war das bei den Hungerzahlen. Da hat die Welternährungsorganisation FAO im Jahr 2012 ihre Methodologie verändert. Die Hungerzahlen, die seit 1996 immer gestiegen sind, fallen bei Anwendung der neuen Methode seit 1990 ständig. (Frankfurter Rundschau, 11.10.2014, S. 24.)

Methodik und Methodologie weisen allerdings noch weitere Gebrauchsweisen auf, die in wissenschaftlichen Zusammenhängen belegt sind. Während man mit $M e$ thodik die Gesamtheit aller zur Verfügung stehenden wissenschaftlichen Ansätze oder Verfahren meint, bezieht man sich mit Methodologie auf die Verfahrenstheorie, wie sie vor allem in Geisteswissenschaften als die Theorie und Lehre der wissenschaftlichen Verfahren vorkommt. Ebenfalls interessant ist der Bezug von $\mathrm{Me}$ thodik und Methodologie auf Lehrfächer. Auch wenn in diesen Zusammenhängen viele kontextuelle, also rein sprachliche Ähnlichkeiten vorhanden sind, bezeichnen sie außersprachlich inhaltlich $\mathrm{zu}$ trennende Lehrfächer, die auch unterschiedlichen Disziplinen angehören (Methodik = Pädagogik/Lehramt vs. Methodologie $=$ Wissenschaftstheorie/Philosophie). Genau hier erfolgt in der allgemeinen Wissenschaftssprache die definitorische Schärfung der Ausdrücke. Dennoch stehen die starken Überlappungen hinsichtlich der oben erwähnten Kontexte stärker im Fokus, sind geläufiger und üben einen gegenseitigen Einfluss aus. Die 
Verwischung semantischer Grenzen in der Alltagssprache führt zu ungenügender Präzisierung in der Wissenschaftssprache oder zu nicht mehr ausreichendem Wissen, dass es in wissenschaftssprachlicher Hinsicht eine genauere terminologische Abgrenzung überhaupt gibt. Das lässt sich u.a. daran erkennen, dass genau diese Ausdrücke immer wieder Gegenstand der universitären Vermittlung wissenschaftlicher Grundlagen sind.

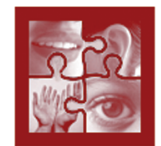

Einführung in die empirischen Methoden der Kultur- und Sozialanthropologie

Ernst Halbmayer

Institut für Kultur- und Sozialanthropologie, Universität Wien

\section{$\underline{2}$ Einige wissenschaftstheoretische Grundlagen der empirischen Sozialforschung}

\subsection{Methoden, Methodik und Methodologie}

Im Deutschen können drei eng verwandte Begrifflichkeiten unterschieden werden, nämlich Methoden, Methodik und Methodologie.

Auf der allgemeinsten Ebene kann man unter Methodik die Gesamtheit der Techniken der wissenschaftlichen Vorgehensweisen bezeichnen. In diesem Sinne kann man etwa von einer Methodik des Evolutionismus, des Diffusionismus, des Funktionalismus $\mathbf{u}^{\top}$ oder Strukturalismus sprechen.

Im Gegensatz dazu kann man auf einer konkreteren Ebene von Methoden in zweifacher Hinsicht sprechen. Einerseits von Methoden der Datenerhebung (Befragung, Beobachtung etc.), andererseits von Methoden der Datenanalyse (Inhaltsanalyse, Diskursanalyse etc.). Innerhalb der Kultur- und Sozialanthropologie wird oft nicht hinreichend zwischen Methodik und den konkreten Methoden unterschieden und z.B. von der Methode der Konstruktion menschlicher Entwicklungslinien im Evolutionismus oder von Kulturkontakten im Diffusionismus gesprochen.

Im Gegensatz zu Methodik und Methode bezeichnet der Begriff der Methodologie die Theorie bzw. Lehre von den wissenschaftlichen Methoden, d.h. zur Methodologie gehören Aussagen und Kriterien,

- welche Methode für bestimmte Anwendungen geeignet ist

- wieso eine bestimmte Methode angewendet wird und keine andere

- warum für bestimmte Probleme eine bestimmte Methode angewandt werden sollte

Oft wird der Begriff der Methodologie auch unpräzise für Methodik verwendet, wie etwa in der Aussage "Diese Untersuchung bedient sich folgender Methodologie."

Erschwerend kommt hinzu, dass die begriffliche Unterscheidung zwischen Methodik und Methodologie in anderen Sprachen, wie z.B. dem Englischen nicht existiert. Der englische Begriff der methodology umfasst Methodik und Methodologie.

Abbildung 3: Beispiel für wissenschaftliche Grundlagenvermittlung von Termini aus: https://ww w.univie.ac.at/ksa/elearning/cp/ksamethoden/ksamethoden-29.html

In der allgemeinen Wissenschaftssprache bzw. in Fachsprachen unterschiedlicher akademischer Disziplinen spielen die Ausdrücke (und Konzepte) Methode und Methodologie seit jeher eine zentrale Rolle. Beide Ausdrücke sind aufeinander bezogen und bilden das Fundament wissenschaftlicher Disziplinen; dies erweist sich allerdings nur dann als tragfähig, wenn wissenschaftliche Verfahrens- 
weisen, also Methode(n) im Rahmen einer Wissenschaftstheorie terminologisch klar definiert und abgegrenzt werden. Entsprechend findet man in einschlägigen allgemeinwissenschaftlichen terminologischen Lexika ${ }^{10}$ auch die Stichwörter $M e$ thode und Methodologie. Die dazugehörigen Erklärungen sind aus zwei Gründen komplex: Einerseits wird die zu berücksichtigende Zeitspanne von der Antike bis heute abgedeckt; andererseits versucht man, das Spektrum möglichst vieler wissenschaftlicher Bereiche zu berücksichtigen. Besonders auf dem Gebiet der Philosophie bemühte man sich seit jeher um eine inhaltlich und terminologisch klare Definition von Methode:

Mit dem Anspruch, die allen M[ethoden] gemeinsamen Züge kenntlich machen zu können, tritt seit der Antike die philosophische M[ethode] auf. Platon kennzeichnet sie [...] als Kunst (-fertigkeit) des argumentierenden Gesprächs, des mit dem Willen zur Verständigung geführten (philosophischen) Dialogs. (Mittelstraß (Hrsg.) 1984: 876)

Seit diesen Anfängen wurde - für ganz unterschiedliche wissenschaftliche Disziplinen - eine Vielzahl unterschiedlicher Methoden entwickelt, erprobt, und definiert. Voraussetzung dieses ,Terminologisierungsprozesses ‘ ist das Vorhandensein einer Methodologie. Sie wird definiert als

die den Wissenschaften als Teil einer allgemeinen Logik der Wissenschaften vorausgehende Lehre von den (wissenschaftlichen) Methoden, als solche Teil der (allgemeinen) Wissenschaftstheorie. (Mittelstraß (Hrsg.) 1984: 887)

Methode(n) und Methodologie sind im Rahmen fachsprachlicher wissenschaftlicher Theoriegebäude also klar umrissene Begriffe von zentraler Wichtigkeit. Stark vereinfacht lassen sich die Zusammenhänge wie folgt auf dem Punkt bringen:

Der Weg des wissenschaftlichen Vorgehens wird als Methode bezeichnet. Vom Standpunkt der heutigen Wissenschaftstheorie aus kann man sagen, daß den einzelnen Wissenschaftsbereichen Methoden zugeordnet sind, so zum Beispiel der Mathematik die deduktive Methode, den Natur- und teilweise Sozialwissenschaften die induktive Methode, den Geisteswissenschaften und ebenfalls teilweise den Sozialwissenschaften die phänomenologische, die hermeneutische, die historische Methode [...]. (Mittelstraß, Jürgen (Hrsg.) 1984: 887)

Methodik wird i.d.R. im Kontext einer Einzelwissenschaft z.B. wie folgt umschrieben: ,wissenschaftstheoretisch die Gesamtheit der Methoden, die im Prozess der Forschung zur Bearbeitung definierter Themen- und Fragestellungen genutzt werden“ (Tenorth \& Rudolf 2007: 506). Als Kollektivum lässt sich Methodik deshalb als Werkzeugkasten verstehen, der alle Instrumente und Anweisungen enthält,

10 Z.B. Seiffert und Radnitzky (1989). 
die zur Bewältigung einer Aufgabe in einem bestimmten Aufgabenbereich nötig sind. Man verwendet den Ausdruck Methodik in dieser Weise häufig im Kontext mehr oder minder fachsprachlicher Kommunikation. In diesem Rahmen fungiert Methodik in erster Linie als (allgemeinsprachennahes) Kollektivum und bestenfalls in zweiter als fachsprachlich näher spezifizierter und definierter Terminus. Entsprechend finden sich auch in der öffentlichen Gebrauchssprache Verwendungen, in denen der Plural Methoden und das Kollektivum Methodik im Rahmen einer Fachdisziplin synonym verwendet werden.

17. Egon Stumpf führte aus: ,Jede Zeit hat ihre künstlerischen Ausdrucksformen. Da unsere Zeit von der Wissenschaft geprägt ist, nehmen wir die Welt durch wissenschaftliche Methoden wahr. Doch die wissenschaftlichen Aussagen sind durch ihre Methodik begrenzt.“ (Fränkischer Tag, 01.05.2012, S. 16, Flugsamen als Geometrie-Form.)

18. Eine theoretische und teils praktische Einführung in die Methodik der Psychotherapien bietet ein Seminarwochenende am 14./15. November. Dabei werden sowohl die gängigen Methoden vorgestellt als auch Wünsche der Teilnehmer berücksichtigt. (Rhein-Zeitung, 30.10.1998, Griechischer Abend.)

Die alltagssprachlichen Einflüsse auf die Wissenschaftssprache werden am Beispiel Methode/Methodologie/Methodik gut sichtbar. Die Rivalität der einzelnen Ausdrücke und deren Vorkommen sowohl in der Alltags- (Methode) wie auch in der Wissenschaftssprache (Methodologie/Methodik) sorgen für das Verschwimmen von semantischen Grenzen in der Alltagskommunikation. Daraus wiederum resultiert der semantische Transfer im Gebrauch der Ausdrücke in die Wissenschaftssprache. Hier aber ist eine Differenzierung nötig, um wissenschaftlich präzise und korrekt Sachverhalte darstellen zu können. Der Mangel an sprachlichem Wissen zeigt, wie auch alltagssprachliche Ausdrücke in wissenschaftssprachlichen Kontexten vermittelt und erlernt werden müssen. Eine sprachliche Vermischung von ähnlichen Ausdrücken wie Methode/Methodologie führt in der allgemeinen Gebrauchssprache i.d.R. nicht zu Fehlinterpretationen, in der wissenschaftssprachlichen Kommunikation kann sie hingegen zu Missverständnissen führen. Anders als am Beispiel unehelich/nichtehelich/außerehelich, bei dem die sprachliche Steuerung in der Verwaltungssprache Einflüsse auf den Gebrauch in der Alltagssprache hat, liegt hier der umgekehrte Fall vor: Der alltagssprachliche Gebrauch hinterlässt Spuren in der Wissenschaftskommunikation. 


\section{Bildungssprachliche Paronyme}

Neben fachsprachlichen und allgemeinwissenschaftlichen Paronymen gibt es auch noch solche Paronympaare, die sich in stilistischer Hinsicht als bildungssprachlich charakterisieren lassen, wobei eine strikte Trennung zwischen den Kategorien mitunter schwierig ist. Es gibt einige Paare, die durchaus als verbreitet gelten, wie z.B. normierte Fachbegriffe (konkav/konvex), aber auch solche, die in Abhängigkeit von Bildungsinhalten bzw. deren Vermittlung durch allgemeine oder höhere Schulbildung im Alltag vorausgesetzt werden können (effizient/effektiv, sensibel/sensitiv, rational/rationell, formell/formal). In der Regel erfolgt die Vermittlung dieses eher abstrakten und für das Schriftdeutsch typischen Inventars über einen sprachsensiblen Fachunterricht. Auch Paare wie ethisch/ethnisch, temporär/temporal, konzeptionell/konzeptuell, patriarchalisch/ patriarchal/patriarchisch, funktionell/funktional, fiktiv/fiktional zählen hierzu. Ihre Verbreitung ist im öffentlichen Sprachgebrauch unterschiedlich ausgeprägt, oftmals sind sie im niedriger frequenten Bereich anzusiedeln. Sie sind häufig fremdsprachlicher Herkunft und ihr Gebrauch ist themen- oder diskursspezifisch. Ihr angemessener Gebrauch setzt einen höheren Kenntnis- oder Erfahrungsstand voraus. Ähnlich wie in der Fachsprache dienen diese Ausdrücke der Präzisierung von bestimmten Konzeptualisierungen, die nötig sind, um komplexe sachliche Sinnzusammenhänge mit sprachlichen Mitteln auszudrücken. Auch um mit bestimmten fach- oder themenspezifischen Inhalten produktiv umgehen zu können, sind Kompetenzen aus dem bildungssprachlichen Register von Bedeutung.

Paronyme, die diesem Register/dieser Varietät angehören, weisen sehr unterschiedliche Verhaltensmuster auf. Bei einigen kommt es zu Verwechslungen, bei anderen geschieht dies kaum. Nicht selten entstehen Verwechslungen auch durch die Bemühung, stilistisch ein sprachliches Register wählen zu wollen, ohne alle dafür erforderlichen sprachlichen und/oder außersprachlichen Informationen zur Verfügung zu haben. Der Gebrauch bildungssprachlicher Paronyme ist stilistisch markiert. Zahlreiche Verwechslungen resultieren auch aus dem individuellen Bemühen um kontextuell stilistische „Reife“.

Gerade solche Ausdrücke sind öfter davon betroffen, sprachlich in enger Nachbarschaft, in koordinierenden Konstruktionen paarformelhaft genutzt zu werden, wie z.B. rational und rationell, effektiv und effizient, fiktiv und fiktional:

19. Resümee: Auch die „Philosophie“ der Verkehrsstruktur sollte rational und rationell sein. (Nordkurier, 21.08.2012, Leser schreiben an die Redaktion.)

20. Entscheidungsprozesse müssen für jeden nachvollziehbar dargelegt werden. Des Weiteren sollte sie von der Politik (Gemeinderat) vorgegebene Ziele und Leitbilder effektiv 
und effizient umsetzen und intern für die Mitarbeiter ein gutes und vertrauensvolles Arbeitsklima aufweisen. (Badische Zeitung, 23.01.2010, S. 33.)

21. Ja, das war wohl das auffallendste auf der Duisburger Filmwoche in diesem Jahr, daß Produktionen gezeigt wurden, die das Genre um fiktive oder fiktionale Elemente erweitern und die Grenze zwischen „echt“ und „falsch“ zum Fließen bringen. (Frankfurter Allgemeine, 13.11.2003.)

Solche koordinierenden Konstruktionen haben eine bestimmte Funktion. Sie werden immer dann genutzt, wenn sprachliche Unsicherheiten in der Abgrenzung beider Ausdrücke ausgeblendet werden sollen, indem semantische Inhalte zusammengefasst werden. Die häufige Nutzung solcher Konstruktionen kann aber auch zu Semantisierungsprozessen führen, die beide Ausdrücke einander semantisch angleichen bzw. einen der Ausdrücke an den anderen anpasst und als Folge dessen synonymisiert. Regelhaftes gemeinsames Vorkommen kann also ein sprachlicher Faktor zur Etablierung von Bedeutungsnähe sein. So auch bei Adjektiven auf -al/-ell: universal/universell, kategorial/kategoriell, original/originell (siehe Storjohann 2010 oder sensibel/sensitiv in Storjohann 2015). Deshalb scheint es nach derzeitigem Kenntnisstand ${ }^{11}$, dass diese Erscheinung bei bildungssprachlichen Paronymen stärker zu beobachten ist als bei alltagssprachlichen Ausdrücken. ${ }^{12}$ Sie sind einerseits stärker von einseitiger oder gegenseitiger Verwechslung betroffen als alltagssprachliche Paronyme und andererseits tendieren sie auch stärker zur Bedeutungsangleichung. Diese These gilt es jedoch, noch weiter zu untermauern.

\section{Ursachen von Verwechslung stilistisch markierter Paronyme}

Sprachliche Unsicherheiten bei Sprecher*innen können von verschiedenen sprachlichen als auch außersprachlichen Faktoren abhängen. Lăzărescu (1995, 1999) beschäftigte sich ausführlich mit Paronymen aus strukturalistischer Sicht und mit einem fremdsprachlich-didaktischen Ansatz. Durch die präskriptive Brille betrachtet ist die Paronymie für ihn eine „Fehlerquelle“ (1999: 17). Er ließ Erklärungen, die außersprachliche Faktoren berücksichtigen, außer Acht, da sie für

11 Rund 150 Paronympaare/-gruppen verschiedenster Kategorien und stilistischer Varietäten wurde bisher ausgewertet.

12 So gibt es keine Belege für koordinierende Konstruktionen wie *halbstündig und halbstündlich, *lebenslang und lebenslänglich oder *farbig und farblich. 
ihn zu den psychologischen Erscheinungen zählen und nicht Teil lexikalischer Erklärungen sind. Aus heutiger Sicht sind kognitiv verankerte Faktoren interessant, weil sie kaum von den sprachlichen Faktoren zu trennen und bei der Konstitution kommunikativer Akte beteiligt sind. Weltwissen, Erfahrung, Wahrnehmung und die Wahl lexikalischer Realisierungen für bestimmte Konzeptualisierungen hängen im Sprachgebrauch eng zusammen.

Dazu nachfolgend einige Beispiele. Neben der erwähnten regelhaften Verwendung in Paarformeln (effektiv und effizient), neben politischer Sprachsteuerung (nichtehelich/unehelich/außerehelich), dem Verschwimmen der Grenzen zwischen Fach- und allgemeiner Gebrauchssprache (etwa durch Einfluss der Werbung) wie bei Parodontose und Parodontitis, ist z.B. auch mangelnde Sachkenntnis eine häufige Ursache von Verwechslung. Generell sind hiermit weniger Bildungsstand oder Bildungslücken seitens der Sprecher`innen gemeint als vielmehr der Mangel an speziellem Wissen, wie z.B. bei der Unterscheidung von Kriminalist und Kriminologe. Kriminalist und Kriminologe sind Bezeichnungen für zwei fachspezifische Berufsgruppen. Personen, die als Kriminalisten arbeiten, sind Beamte oder Angehörige der Polizei und ermitteln bei Straftaten. U.a. ermitteln sie Straftäter, sichern Spuren an Tatorten, decken Verbrechen auf und versuchen sie zu verhindern. Ihr Beschäftigungsfeld ist die Kriminalistik. Personen, die dagegen als Kriminologen bezeichnet werden, beschäftigen sich theoretisch wissenschaftlich mit der Lehre der Kriminologie. Sie erforschen und vermitteln Muster, Arten und Charakteristika von Verbrechen. Auch wenn es Überschneidungen im Berufsfeld gibt, handelt es sich um zwei verschiedene Berufsgruppen. Die Ausdrücke sind daher nicht bedeutungsgleich. Dennoch kommt es gelegentlich zu Verwechslungen, dabei wird die Bezeichnung Kriminologe fälschlicherweise für die Bezeichnung Kriminalist verwendet (siehe Beispiel 22).

22. In anderen Ländern bereits eingesetzt, führte im März 1903 der Chef der Dresdner Kriminalpolizei, Paul Koettig, als erster deutscher Fahnder den Fingerabdruck zur Verbrechensbekämpfung ein. Seither sind die feinen Spuren für die Kriminologen zur Identifizierung von Tätern, aber auch von Opfern unentbehrlich. (Leipziger-Volkszeitung, 27.03.2003, S. 4.)

Ein weiterer Fall ist die gegenseitige Verwechslung von Ausdrücken wie konkav und konvex, die aufgrund ähnlicher Referenz (,gewölbt') und gleicher Bezugswörter (z.B. Spiegel, Linse, Gläser) fehlerhaft verwendet werden. Bei diesen Ausdrücken (auch Stalagmit/Stalaktit) sind sich Sprecher*innen durchaus bewusst, dass sich zwei Ausdrücke gegensätzlich gegenüberstehen und zwei eng miteinander verbundene Konzepte benennen. Die richtige Zuschreibung zwischen Konzept und Lexem stellt hier das Problem dar. Die Verwechslungen sind eher außersprachlicher Natur, entstehen also weniger aufgrund semantischer Un- 
genauigkeiten. Für den korrekten Gebrauch der beiden Adjektive existieren Merkhilfen.

23. Lange Zeit glaubte ich, dass Eselsbrücken so heißen, weil sie für die sind, die so dumm sind, wie man es dem Langohr nachsagt. [...] Mir haben diese Gedächtnisstützen immer gute Dienste erwiesen. Einmal im Kopf verschwinden sie nämlich nie wieder. [...] Wer sich gemerkt hat, dass der Podex konvex ist, wird konvex und konkav nie mehr verwechseln. (Nordkurier, 08.03.2008, Damit die Esel ankommen.)

Ein weiterer außersprachlicher Faktor für Verwechslung könnte auch der sogenannte „bathtub effect“ sein. Dieser von Aitchison (1989) geprägte Terminus bezieht sich auf die psycholinguistische Beobachtung, der zufolge es Befragte beim Erinnern eines Ausdrucks (oder eines Namens) einfacher finden, sich an den Anfang oder das Ende zu erinnern, aber nicht an das Mittelstück. ${ }^{13}$ Diese Beobachtung könnte sich auch auf die perzeptuelle Salienz von Paronymen beziehen. Ähnlich klingende Ausdrücke werden deshalb verwechselt, weil sie über die gleiche Anzahl von Silben oder einen gleichlautenden Anklang verfügen und dabei auf ähnliche Konzepte referieren. Der bathtub effect ist zwar Inhalt psycholinguistischer Forschungen, deutschsprachige Paronyme hingegen sind es nicht. In welchem Umfang der bathtub effect eine größere Rolle bei sprachlichen Unsicherheiten mit verwechselbaren Wörtern spielt, ist daher bislang unbekannt. Die rein lautliche Nähe gilt dennoch als eine typische Fehlerquelle, z.B. bei ethisch/ethnisch oder launig/launisch. Diese werden nicht aufgrund semantischer Ähnlichkeiten verwechselt. Die Adjektive weisen nämlich keinerlei semantische Gemeinsamkeiten auf. Im Korpus zeigt sich dabei die stärkere Tendenz, den Ausdruck ethisch anstelle von ethnisch zu nutzen, z.B. ethische Herkunft, statt ethnische Herkunft oder ethische Minderheiten, statt ethnische Minderheiten. Bei solchen Verwechslungen handelt es sich um Fehlentscheidungen bei der Wortwahl.

24. Für die Mitarbeiter (2001: 5.771) gibt es ein Car-Sharing-System und günstige Konditionen beim Fahrradkauf. Auf Frauenförderung und die Förderung sozialer und ethischer Minderheiten wird in diesem Unternehmen großer Wert gelegt. (die tageszeitung, 13.05.2002, S. 9.)

25. „Steht es uns zu, den Wert menschlichen Lebens zu beurteilen nach den Kosten, die es der Gesellschaft verursacht?“ Die Frage nach ethnischen Normen, Gesprächsrunden mit Eltern von behinderten Kindern und sonstigen Betroffenen, ein Besuch im Altenheim waren Bestandteil der „Woche für das Leben“ in Jeckenbach. (Rhein-Zeitung, 21.06.1997, Behinderte.)

13 In Anlehnung an eine Person in der Badewanne, ist dabei der Kopf am prominentesten, der Rumpf ist nicht sichtbar und die Füße sind weniger sichtbar als der Kopf. 
Im Falle von sensitiv und sensibel lassen sich neben lautlicher und semantischer Ähnlichkeit zusätzlich Einflüsse der englischen Sprache ausmachen, die über Übersetzungsfehler zu Unsicherheiten im Gebrauch von sensitiv geführt haben und damit einen Bedeutungswandel auslösten (Details dazu in Storjohann 2015). ${ }^{14}$

Auch falsche morphologische Analogien wie Diplomatie/Diplomatik führen zu fehlerhaften Kontexten. Die Ausdrücke Diplomatie und Diplomatik beziehen sich auf unterschiedliche Konzepte und sind nicht bedeutungsgleich. Diplomatie bezieht sich auf eine Fähigkeit oder auf eine Personengruppe und wird überwiegend in politischen Kontexten verwendet. Diplomatik hingegen ist der Fachausdruck zur Bezeichnung der Urkundenforschung. Dieser Ausdruck ist äußerst selten in der öffentlichen Gebrauchssprache belegt. Dennoch kommt es vereinzelt zu Verwechslungen, bei denen Diplomatik im Sinne von Diplomatie genutzt wird.

26. Kissingers breit und differenziert angelegtes, auf eine Riesenfläche projiziertes Portrait Chinas lässt sich unter verschiedenen Aspekten lesen. Es ist eine äußerst intensive und faktenreiche, stets um Analyse und Erkenntnis bemühte Studie zur chinesischen Geschichte und den prägenden Mentalitäten - vom zweiten vorchristlichen Jahrtausend bis zur unmittelbaren Gegenwart. Es ist ein Lehrbuch der Diplomatik. Eine Einführung in die Mysterien, Raffinessen und Banalitäten der Staatskunst. (Nürnberger Zeitung, 15.12.2011, S. 28.)

Es wäre hier möglich, dass -ik als Suffix zur Kennzeichnung eines (Wissenschafts-)Gebiets, einer Disziplin oder eines Bereichs (analog zu Germanistik, Orientalistik) genutzt wird, um aus Diplomatie einen Ausdruck zu schaffen, der sich auf die Gesamtheit oder die Lehre der Diplomatie beziehen kann. Dass der selten belegte Fachbegriff Diplomatik dabei schon existiert und einen völlig anderen Bereich bezeichnet, zeugt davon, dass die Kenntnis von der Existenz dieses Ausdrucks fehlt.

Als vorletzter Punkt sei hier noch die rein konzeptuelle Nähe z.B. zwischen autoritär/autoritativ genannt, die ebenfalls zu Unsicherheiten und Verwechslungen führt. Beide Adjektive gehen auf das Nomen Autorität zurück und beziehen sich auf Führungs- und Erziehungsstile. Mit autoritär bezeichnet man einen Stil von oben (einer Autorität) herab, anordnend, keinen Widerstand duldend und ohne Rücksicht auf die Bedürfnisse anderer nehmend. Mit autoritativ bezieht man sich im Allgemeinen auf einen Stil, der Richtlinien seitens einer Autorität vorgibt,

14 Sensibel/sensitiv sind falsche Freunde mit den englischen Entsprechungen sensible/sensitive und keineswegs Bedeutungsäquivalente. 
aber das Interesse auf Selbstverwirklichung Anderer wahrt und die Wünsche Anderer unterstützt. Nicht immer wird diese Unterscheidung sprachlich klar genug getroffen. Besonders bei der Verwendung von autoritativ kommt es zu Verwechslungen mit autoritär, sowohl im Sinne von politisch diktatorisch als auch in Bezug auf Verhalten und Umgangston, der als anordnend interpretiert werden kann. Umgekehrt wird autoritär nicht im Sinn von autoritativ verwendet.

27. Die jahrhundertelang praktizierte obrigkeitsstaatliche Antwort, die ein kirchlich definiertes Gemeinwohl unter Verzicht auf Demokratie und Partizipation autoritativ ,von oben' durchsetzen wollte, ist dabei ebenso unbrauchbar wie die klassische Lösung des politischen Liberalismus. (Frankfurter Rundschau, 12.10.1999, S. 23.)28. Elias Canetti hat daran erinnert, dass der Befehl älter als die Sprache sei. Er wirke so autoritativ wie eine göttliche Macht, erlaube keinen Widerspruch und komme von außen. (die tageszeitung, 01.09.2007, S. 9.)

Die Ursachenforschung, die der Frage nachgeht, was konkret Verwechslung auslöst, gibt wichtige Indikatoren zur Aufdeckung von Prinzipien des Bedeutungswandels und Erklärungen für das Entstehen neuer Semantisierungsprozesse. In Bezug auf die Paronymie beginnen wir gerade erst mit dieser Forschung. So können die aufgeführten Erklärungsversuche nicht den Anspruch auf Vollständigkeit erheben, sie skizzieren vielmehr beispielhaft einige typische Muster.

\section{Ausblick}

Sprachgebrauchsorientierte, deskriptive Paronymforschung ist in der Lage, die Vielfalt und Dynamik eines Phänomens zu beleuchten, das aus korpuslinguistischer oder kognitiv-semantischer Sicht bisher auf wenig Interesse gestoßen ist. Die Paronymie ist nicht nur ein lexikalisches Phänomen, sondern auch ein konzeptuelles. Sie ist vielschichtig und ihre Komplexität drückt sich in zahlreichen Schnittstellen aus, die bisher weitgehend unberücksichtigt blieben. Empirische Verfahren ermöglichen uns heute, neu an diese Fragestellungen heranzutreten und eine objektivere Beschreibung zu gewährleisten. Im Rahmen korpussemantischer Analysen lassen sich Strukturen und Verwendungsweisen aufdecken, die Paronyme in unterschiedlichen kommunikativen Situationen und Diskursen zeigen. Erste korpussemantische Betrachtungen sind in Storjohann (Hrsg.) (2019) dargelegt. Das Spannungsfeld zwischen alltags- und fachsprachlichen Verwendungen leicht verwechselbarer Ausdrücke genauer zu skizzieren, war das Ziel dieses Beitrages. Dabei wird eine Dynamik und gegenseitige Einflussnahme einzelner Verwendungen deutlich. Diese Dynamik wird sowohl durch sprachliche als auch durch außersprachliche Faktoren in unterschiedlichem Maße bei der Kommunikation angesto- 
ßen. Dies führt mal zu Fehlern, mal zu Verwechslungen und manchmal zu semantischen Anpassungen. Die Auswirkungen sind höchst unterschiedlich: Sie variieren von Wortpaar zu Wortpaar, unterscheiden sich innerhalb morphologischer Muster und folgen keinem vorgegebenem Schema. Die richtige Beschreibung solcher Fälle birgt einige Herausforderungen, man denke etwa an die Vermittlung solcher Lexeme im Fremdsprachenunterricht oder deren Erfassung in der Fachwortdidaktik (Roelcke 2015) bzw. in der Lexikografie (Storjohann 2019).

Bisher fehlt eine integrierte Darstellung der kognitiven und linguistischen Prinzipien, die Paronymie ausmachen. Dazu bedarf es einer Theorie, die empirisch orientiert ist und den aktuellen Gebrauch der Paronyme untersucht. Die Ergebnisse müssen mit Ansätzen der kognitiven Semantik, aber auch der Psycholinguistik abgerundet werden, um sich dem Phänomen von allen Seiten zu nähern. Das Projekt ,Paronymwörterbuch` geht einen ersten wichtigen Schritt in diese Richtung. Es beschreibt leicht verwechselbare Ausdrücke korpusgestützt und dokumentiert seine Ergebnisse in einem neuen, konzeptuell ausgerichteten Nachschlagewerk, in kontrastiv angelegten, dynamischen und deskriptiven Wörterbuchartikeln. Es versucht, kommunikativen paronymen Gebrauch angemessen zu beschreiben, die Differenzen zu fachsprachlichen Verwendungen aufzuzeigen und bildungssprachlichen Gebrauch zu markieren, aber auch Tendenzen im Wandel oder im fehlerhaften Gebrauch zu erklären. Lexikologisch setzt sich das Projekt mit zahlreichen Forschungsfragen auseinander, die den Blick auf die Paronymie verändern werden.

\section{Literatur}

Aitchison, Jean. 1987. Words in the Mind: An Introduction to the Mental Lexicon. Oxford/Cambridge (MA): Blackwell.

Berg, Tanja \& Susanne Sonnenfeld (Hrsg.). 2012. Die Entwicklung des Sorgerechts der Mütter nichtehelicher Kinder in Deutschland vom Inkrafttreten des BGB bis heute. Beiträge aus dem Fachbereich Rechtspflege Nr. 02/2012, S. 41, (https://opus4.kobv.de/opus4-hwr/ frontdoor/index/index/docld/903), abgerufen am 09.01.2019.

Ehlich, Konrad. 2000. Deutsch als Wissenschaftssprache für das 21. Jahrhundert. German as a Foreign Language (GFL) 1/2000. 47-63. (http: //www.gfl-journal.com).

Lăzărescu, laon. 1999. Die Paronymie als lexikalisches Phänomen und die Paronomasie als Stilfigur im Deutschen. Bukarest: Anima.

Lăzărescu, Ioan. 1995. Deutsche Paronyme. Grazer Linguistische Studien 4. 85-93.

Mell, Ruth, Ulrich Schnörch \& Petra Storjohann. 2019. Empirische, lexikologische Annäherung an die Paronymie im Deutschen. In Storjohann, Petra (Hrsg.), Paronymie im deutschen Sprachgebrauch. Sonderheft Deutsche Sprache 1/2019. 53-67.

Mittelstraß, Jürgen (Hrsg.). 1984. Enzyklopädie Philosophie und Wissenschaftstheorie. Mannheim, Wien \& Zürich: Bibliographisches Institut \& Wissenschaftsverlag. 
Müller, Markus. 2015. Sprachliches Rollenverhalten. Korpuspragmatische Studien zu divergenten Kontextualisierungen in Mündlichkeit und Schriftlichkeit. Berlin \& Boston: Walter de Gruyter.

Roelcke, Thorsten. 2010. Fachsprachen. 3. Auflage. (= Grundlagen der Germanistik 37). Berlin: Erich Schmidt.

Roelcke, Thorsten. 2015. Besondere Wörter II: Fachwörter, Termini. In Hass, Ulrike \& Petra Storjohann (Hrsg.), Handbuch Wort und Wortschatz (= Handbücher Sprachwissenschaft 3), 371-393. Berlin \& Boston: de Gruyter.

Schnörch, Ulrich. 2015. Wie viele Paronympaare gibt es eigentlich? Das Zusammenspiel aus korpuslinguistischen und redaktionellen Verfahren zur Ermittlung einer Paronymstichwortliste. Sprachreport 2015/4. 16-26.

Schnörch, Ulrich. 2019. Kookkurrenzanalyse und Vergleich: Überlegungen zur Methodenanwendung bei der lexikografischen Beschreibung von Paronymen. In Storjohann, Petra (Hrsg.), Paronymie im deutschen Sprachgebrauch. Sonderheft Deutsche Sprache 1/2019. 12-24.

Seiffert, Helmut \& Gerard Radnitzky (Hrsg.). 1989. Handlexikon zur Wissenschaftstheorie. München: Ehrenwirth.

Storjohann, Petra. 2010. Synonyms in corpus texts: Conceptualisation and construction. In Storjohann, Petra (Hrsg.), Lexical-Semantic Relations. Theoretical and practical perspectives. (= Lingvisticae Investigationes Supplementa 28), 69-94. Amsterdam: Benjamins.

Storjohann, Petra. 2015. Was ist der Unterschied zwischen sensitiv und sensibel? Zeitschrift für Angewandte Linguistik 62. 99-122.

Storjohann, Petra (Hrsg.). 2019. Paronymie im deutschen Sprachgebrauch. Sonderheft Deutsche Sprache 1/2019.

Storjohann, Petra. 2019. Paronyme - Dynamisch im Kontrast. Ein kognitiv ausgerichtetes, multifunktionales, dynamisches Nachschlagewerk. In Storjohann, Petra (Hrsg.), Paronymie im deutschen Sprachgebrauch. Sonderheft Deutsche Sprache 1/2019. 82-94.

Teichmann, Mareike. 2019. SOM und CNS als korpuslinguistische Methoden zur Analyse von Paronymen am Beispiel technisch/technologisch. In Storjohann, Petra (Hrsg.), Paronymie im deutschen Sprachgebrauch. Sonderheft Deutsche Sprache 1/2019. 25-41.

Tenorth, Heinz-Elmar \& Rudolf Tippelt (Hrsg.). 2007. Lexikon Pädagogik. Weinheim \& Basel: Beltz. 